\title{
Tianeptine and paroxetine in major depressive disorder, with a special focus on the anxious component in depression: An international, 6-week double-blind study
}

Jean Pierre Lepine', Carlo Altamura², Marc Ansseau ${ }^{3}$, Jose Luis Ayuso Gutierrez ${ }^{4}$, Istvan Bitter ${ }^{5}$, Malcolm Lader ${ }^{6}$ and Lionel Waintraub ${ }^{7}$

${ }^{1}$ Service de Psychiatrie, Hôpital Fernand Widal, 200, rue du Faubourg St Denis, 75745 Paris Cedex 10, France

2Università degli Studi di Milano, Cattedra di Psichiatria, Ospedale "L. Sacco", Via G.B.Grassi 74, 20157 Milan, Italy

${ }^{3}$ Service de Psychiatrie, C.H.U. du Sart Tilman, B. 35, B-4000 Liège 1, Belgium

${ }^{4}$ Servicio de Psiquiatria, Hospital Clinico de San Carlos, Martin Lagos, s/n, 28040 Madrid, Spain

${ }_{5}^{5}$ Department of Psychiatry and Psychotherapy, Semmelweis Orvostudomanyi Egyetem, Ballassa u.6,

H-1083 Budapest, Hungary

${ }^{6}$ Clinical Pharmacology Section, Institute of Psychiatry, Decrespigny Park, Denmark Hill, London SE5 8AF, $U K$

${ }^{7}$ Service de Psychiatrie, Centre Hospitalier Paul Brousse, 12 avenue Paul Vaillant Couturier, 94804 Villejuif, France

KEYWORDS: anxious symptoms; major depressive disorder; paroxetine; tianeptine

\section{ABSTRACT}

Tianeptine (37.5 mg/day) and paroxetine (20 mg/day) were compared in a population of depressive patients without past or current history of co-morbid anxiety and/or important anxiolytic treatment. In a 6-week, double blind trial, the special focus was on anxious symptoms.

Both drugs showed good efficacy on depressive symptomatology, assessed with MADRS and HDRS, but no difference was detected between tianeptine and paroxetine, for any assessment criterion. Despite the choice of selected depressive patients, without any co-morbid anxious disorder, anxiety scale scores at inclusion (HAMA and BAS) were appreciable but correlated poorly with depressive scores. Both tianeptine and paroxetine improved the apparent anxious component in depression. Tolerability of both drugs was good, although significantly better with tianeptine.

Thus tianeptine and paroxetine are effective and safe treatments for major depression and may also act directly on the anxious component of the psychopathology. 


\section{Introduction}

Tianeptine is an atypical tricyclic compound, for which antidepressant efficacy has been well established, particularly in comparison with amitriptyline (Lôo et al., 1988; Guelfi et al., 1989), imipramine (Cassano et al., 1996), fluoxetine (Alby et al., 1993; Lôo et al., 1999) and placebo (Cassano et al., 1996; Costa e Silva et al., 1997; Dalery et al., 2001). Preclinical and clinical studies suggest that it may have some anxiolytic properties in depressed patients. Paroxetine is also a well-known antidepressant, displaying anxiolytic properties (Boyer and Feighner, 1992; Foster and Goa, 1997). It was, therefore, interesting to compare these drugs both as regards their antidepressant efficacy, and their respective actions on the anxious component of depression.

Depression and anxiety are frequently associated and have in common many symptoms and signs, as shown in epidemiological, co-morbidity studies (Lépine, 1994; Wittchen et al., 1994; Kessler et al., 1996; Maier and Falkai, 1999). Phenomenological studies have shown that the incidence of anxious symptoms in depression is high, namely $62-98 \%$ of depressive patients (Hamilton, 1989; Kuhs, 1991). Moreover, some authorities have suggested that a subtype of depression characterised by anxiety should be recognised. Some studies have reported a poorer outcome and response to antidepressant medication for this syndrome than for 'pure' depression (Andreasen et al., 1980; Kravitz et al., 1989). However, current classifications do not as yet include a separate category of depression based on anxiety symptoms, as many other studies have failed to show specific characteristics for these 'anxious depressions', especially with respect to prognosis (Joffe et al., 1993). But, beside specific depressive disorders and anxiety disorders, a mixed anxiety/depressive disorder may exist (Sartorius et al., 1996; Boulenger et al., 1997; Wittchen et al., 2001) and diagnostic criteria for clinical research are provided in the DSM-IV classification (American Psychiatric Association, 1994).

The ICD-10 (World Health Organization, 1993) classification acknowledges its existence and apparently high incidence. This symptomatic overlap may also be observed in the assessment tools used for the measurement of anxiety and depression (Watson et al., 1995a,b). The measurement of depressive symptom patterns is quite simple from a methodological point of view. However, measurement of efficacy on the anxious symptoms of depression leads to difficult methodological problems. In fact, while depression and anxiety clearly differ as symptoms, it is difficult to distinguish the syndromes or the mental disorders of which they are characteristic features. The main problem stems from the fact that statistical correlations between depression and anxiety rating scales in different clinical and non-clinical populations are often surprisingly high (Wetzler and Katz, 1989; Clark and Watson, 1991; Watson et al., $1995 a, b)$. Consequently, the combined use of depression and anxiety tools is not necessarily a valid approach for distinguishing an anxiolytic effect from an antidepressant one (Wetzler and Katz, 1989): multivariate analysis may provide more specific depression and anxiety instruments (de Bonis et al., 1991; Martinsen et al., 1989).

Further, to the question of the relationship between depression and anxiety from a nosological and diagnostic point of view, treatments for depression should also be found effective in 
relieving anxious symptoms in depressive patients, as they are common, painful and potentially associated with a higher suicide risk (Fawcett, 1997; Maier and Falkai, 1999).

The present study had two aims. The first was to compare the antidepressant efficacy of tianeptine to that of paroxetine in the treatment of patients with a major depressive episode. Safety data were also recorded in order to compare the tolerability of the compounds.

The second aim was to evaluate the effectiveness of tianeptine versus paroxetine on anxiety in these patients. Since anxious symptoms seem so frequent in patients with any depressive disorder, this study aimed to evaluate major depressive episodes as 'pure' as possible, and not in a selected sample of particularly anxious, depressed individuals. The preliminary correlations observed between depression and anxiety scales suggested that this evaluation could be made either directly through the anxiety scales used in the study, or after factorial analysis of all the assessment tools used in the study, with post hoc identification of anxiety-related clusters.

The present publication presents the efficacy results of the study (antidepressant and anxiolytic actions) and tolerability of the two compounds. Specific methodological aspects of this trial, including the possible overlap in assessment tools for measurement of anxiety and depression and correlations between antidepressant and anxiolytic effects, will be presented separately in detail elsewhere.

\section{Methods}

This multicentre, international, double-blind study was conducted in eight countries: France (20 centres), Belgium (seven centres), Spain (five centres), Hungary (four centres), United Kingdom (three centres), Italy (three centres), Switzerland (one centre) and Portugal (one centre). All investigators were experienced psychiatrists except in the United Kingdom (some investigators were general practitioners with a special training and interest in psychiatric disorders).

The trial was conducted according to the principles in the Declaration of Helsinki and in its latter revisions, and to the rules of European Good Clinical Practices. The initial protocol, the single amendment (for Hungary only) and consent forms in national languages were submitted either to national or local ethics committees, according to national legislation. All patients gave written consent for participation in the study. The clinical part of the study was conducted between November 1997 and May 1999.

\section{PATIENT SELECTION}

Male or female, in- or outpatients, aged from 18 to 65 years, fulfilling DSM-IV criteria for Major Depressive Disorder (single episode or recurrent, severity moderate to severe, without psychotic features, with or without melancholic features and, for recurrent disorders, with or without seasonal pattern and interepisode recovery), were eligible for the study.

To be selected, patients had to present at D7 a minimum severity score of 25 on the Montgomery-Asberg depression rating scale (MADRS) and of nine on the unidimensional subscale of the Hamilton depression rating scale (HDRS, items 1, 2, 7, 8, 10 and 13). These 
severity criteria also had to be present at inclusion, and those patients with a decrease of $30 \%$ or more on MADRS or HDRS global scores were excluded.

Patients fulfilling DSM-IV criteria for other Major Depressive Disorders: Bipolar I or II Disorders (past or present), dysthymic disorder, cyclothymic disorder, mixed anxio-depressive disorder, recurrent brief depressive disorder, schizophrenia or any other acute or chronic psychosis (past or present) and patients whose present disorder was due to a general medical condition or to a substance were not eligible for the study.

Particular interest was focussed on any history of anxious disorders: patients with a lifetime diagnosis (DSM-IV criteria) of anxiety disorder - panic disorder, agoraphobia, specific phobia, social phobia, obsessive-compulsive disorder or acute stress disorder - except generalised anxiety disorder were excluded from the trial. Patients having presented any diagnosis of 'depression plus anxiety' or generalised anxiety disorder within the last year could not be included either. Similarly, special attention was paid to previous anxiolytic treatments. Treatments with a benzodiazepine during the last 2 weeks, or for more than 1 month during the last 6 months, or at a daily dose equal to or greater than an equivalent of $15 \mathrm{mg}$ of diazepam on a lifetime period, were also non-inclusion criteria.

In addition, the following patients were excluded: non-responders to a previous treatment with paroxetine or tianeptine, those defined as non-responders for the current depressive episode (at least two successive well-conducted treatments), any considered at risk of suicide, those incapable of complying with the requirements of the protocol, those with any severe or uncontrolled somatic disease (investigator's judgement), a history of alcohol or drug abuse or dependence, pregnant or breast feeding women, women of child-bearing potential without effective contraception and any who had participated in another clinical study within the last three months or had previously been included in the present study. Patients who had recently ( $<3$ months) initiated or altered treatment with thyroid hormones or, $\beta$-blockers, requiring electro-convulsive therapy or antiepileptic treatment or any CNS-active drug during the study period (such as corticosteroids, ACTH, central $\alpha$-compounds, reserpine, methyldopa or sumatriptan) could not be recruited into the study.

Patients also could not be included if they had been treated with a depot neuroleptic less than 2 months previously, with fluoxetine, non-selective MAOI, lithium, valproate or carbamazepine during the previous month or with any other antidepressant drug during the week before the trial.

\section{STUDY DESIGN}

At selection visit (D7), after giving written, informed consent, patients underwent a complete medical and psychiatric evaluation, with verification of DSM-IV criteria for major depression and MADRS and HDRS assessments. Self-rating scales (severity of depression questionnaire - QSD; hospital anxiety and depression scale - HAD; mood and anxiety symptom questionnaire - MASQ and visual analogue scales - VAS) were also used at selection.

Selected patients then entered a 7-day, run-in, placebo period, which could be shortened to 3 days, depending on the clinical status of the patients. 
Patients were also evaluated at inclusion (D1), during the double-blind treatment period (D3, D7, D14, D28 and D42) and 7 days after treatment cessation (post visit). The interval between visits had to be adhered to, within a range of \pm 3 (D7 and D14) or 6 days (D28 and D42).

After the D42 visit, depending on the patient's condition, the investigators could either stop the treatment (with a post visit 7 days after for evaluating possible withdrawal symptoms) or continue for up to 6 months (optional period). No evaluation was made during the optional period, except for pharmacovigilance data. At the end of the 6-month optional period, a postvisit could be made but was not mandatory.

The main efficacy criterion was the MADRS global score (extracted from the comprehensive psychiatric rating scale - CPRS, except at the selection visit). Other efficacy criteria were observer rating scales: HDRS, HAMA, CPRS (out of which was extracted the brief anxiety scale BAS), the clinical global impression (CGI) and self-rating scales (QSD, HAD, MASQ and six VAS).

Tolerability was assessed by monitoring of adverse events and by using the Arbeitsgemeinschaft für Methodik und Dokumentation Psychoatrischer befunde scale (AMDP-5). The supplement Check Lists d'Evaluation des Symptomes Somatiques (CHESS supplement) was used at the post visit in order to look for possible withdrawal syndromes. Compliance (including morning compliance) was assessed at each visit throughout the study by counting the remaining capsules returned by the patients.

\section{ASSESSMENT TOOLS}

In order to assess accurately the symptom levels, both depression (MADRS and HDRS) and anxiety scales (BAS and HAMA) were used as assessment tools (with MADRS as main efficacy criteria). However, these scales are limited in that they do not evaluate all possible aspects of anxiety and depression but only selected dimensions. Moreover, the anxiety scales have been designed to evaluate anxiety in anxious patients, and their validity in depression can only be assumed. Therefore, a general psychopathology scale was incorporated in order to assess other possible dimensions. For practical reasons, CPRS was chosen as the general psychopathology scale, as it includes both MADRS and BAS. The protocol also stated the intention to study independence between anxiety and depression scales by assessing correlation at inclusion. Other assessment tools used were CGI, as a simple and reliable general scale, and self-evaluation scales (QSD, HAD, MASQ and visual self-rating scales) for evaluating any possible discrepancies.

\section{STATISTICAL METHODOLOGY}

Analysis of the efficacy data was performed both on the intention to treat population (ITT: all patients with at least one evaluation under treatment) and on the per protocol population (PP: all completed patients without major deviation, according to the study protocol). The analysis was done at end-point for the ITT population and visit by visit for the PP population.

The analysis of safety data used all patients who had taken at least one dose of randomised treatment. 


\section{RESULTS}

\section{PATIENTS}

Across the 58 active centres, 366 patients were selected and 327 included (FRA: 146, HUN: 55, GBR: 39, BEL: 38, ESP: 21, CHE: 15, ITA: 9, POR: 4), 162 in the tianeptine group and 165 in the paroxetine group. Out of these, 16 (eight in each group) were considered to deviate markedly from the inclusion criteria (major deviation).

Out of the 269 patients who completed the study up to D42, 133 were in the tianeptine group and 136 in the paroxetine group, and six were considered to present a major deviation $(T=2, P$ $=4)$. The 58 premature withdrawals split equally between each group $(T=29, P=29)$. The intent-to-treat (ITT) population included 317 patients $(T=157, P=160)$ and the per-protocol (PP) population comprised 256 patients $(T=127, P=129)$.

\section{COMPARABILITY AT BASELINE}

Comparability at baseline between treatment groups was assessed, both for demographic variables (Table 1) and for evaluation criteria (Table 2). The only statistically significant difference between treatment groups was for the number of previous depressive episodes.

Table 1. Comparability at baseline (demographic and clinical criteria)a

\begin{tabular}{lcc}
\hline Criteria & Tianeptine $(n=162)$ & Paroxetine $(n=165)$ \\
\hline Age (yr) & & \\
Mean \pm S.D. & $41 \pm 10$ & $42 \pm 12$ \\
Range & {$[19-65]$} & {$[18-64]$} \\
Sex ratio (\%): M/F & $27 / 73$ & $22 / 78$ \\
Hospitalised patients (\%) & 17 & 20 \\
Previous psychotropic treatment (\%) & 67 & 68 \\
Single episode, moderate (\%) & 30 & 30 \\
Single episode, severe (\%) & 8 & 10 \\
Recurrent depression, moderate (\%) & 42 & 41 \\
Recurrent depression, severe (\%) & 20 & 19 \\
Number of previous episodes & $2.2 \pm 1.7$ & $2.8 \pm 2.7$ \\
(for recurrent patients only)* & & 37 \\
DSM-IV criteria for melancholia (\%) & 40 & \\
\hline
\end{tabular}

aAll differences NS except ${ }^{*}: p=0.034$.

Table 2. Comparability at baseline (ITT population)a

\begin{tabular}{lcc}
\hline Criteria & Tianeptine $(n=157)$ & Paroxetine $(n=160)$ \\
\hline & & \\
MADRS global score & $31.5 \pm 4.9$ & $34.4 \pm 4.9$ \\
HDRS global score & $27.4 \pm 4.7$ & $27.1 \pm 4.6$ \\
HAMA global score & $24.4 \pm 7.2$ & $24.1 \pm 7.2$ \\
BAS global score & $18 \pm 6.4$ & $18.4 \pm 6.6$ \\
\hline
\end{tabular}

aAll differences NS. 
Table 3. Evaluation of efficacy at end point (ITT population)a

\begin{tabular}{lcc}
\hline Criteria & Tianeptine $(n=157)$ & Paroxetine $(n=160)$ \\
\hline & & \\
MADRS global score & $13.5 \pm 10.3$ & $13.3 \pm 10.8$ \\
HDRS global score & $12.7 \pm 8.5$ & $12.4 \pm 8.7$ \\
HAMA global score & $11.8 \pm 8.5$ & $11.6 \pm 8.5$ \\
BAS global score & $8.5 \pm 6.5$ & $8.9 \pm 7.2$ \\
\hline
\end{tabular}

aAll differences NS.

\section{CLINICAL EFFICACY}

Depression scales. In the ITT population, mean MADRS and HDRS global scores decreased significantly between inclusion and end point $(p<0.001)$. None of these scales differed significantly between groups (Table 3).

The mean MADRS global score (main criterion) decreased between D1 and end point from 31.5 to 13.5 in the tianeptine group and from 31.4 to 13.3 in the paroxetine group ( $p=0.826$ at end point).

Response to treatment, defined as at least a 50\% decrease in inclusion score, was high with both treatments (more than 60\%), without significant differences between groups, either on MADRS ( $T=96$ [61\%], $P=108$ [68\%], $p=0.930)$ or HDRS ( $T=96$ [61\%], $P=105$ [66\%], $p=0.915$ ). All these results were confirmed in the PP population. No significant difference was observed at D42 and time course evolutions were very similar between groups (Figures 1 and 2).

Anxiety scales. In the ITT population, mean HAMA and BAS global scores also decreased significantly between inclusion and end point $(p<0.001)$. No significant difference between groups was observed (Table 3 ). These results were confirmed in the PP population. No significant difference was observed between groups at D42 and, again, the time courses were very similar (Figure 3).

Other scales. Global assessments (CGI) and data from the different self-rating scales (QSD/R, HAD depression, HAD anxiety, six sub-scales of MASQ and six VAS) confirmed these observations. None of these measures showed any significant difference, with, generally, very similar results between groups.

\section{MADRS scores}

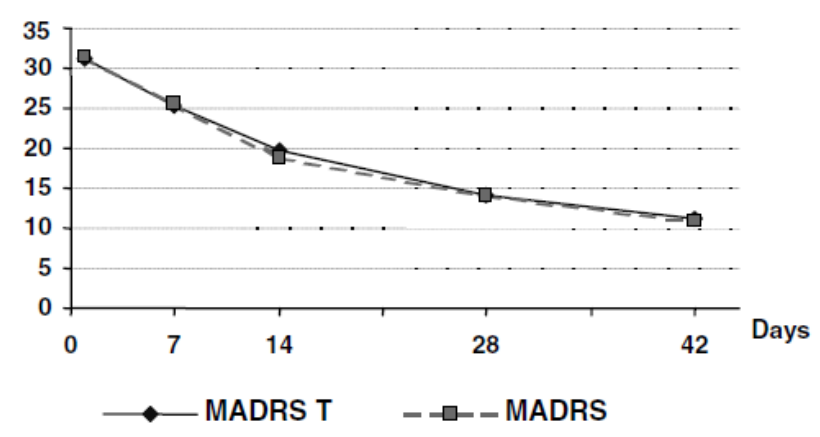

Figure 1. Evolution of MADRS global score (PP population) 


\section{HDRS scores}

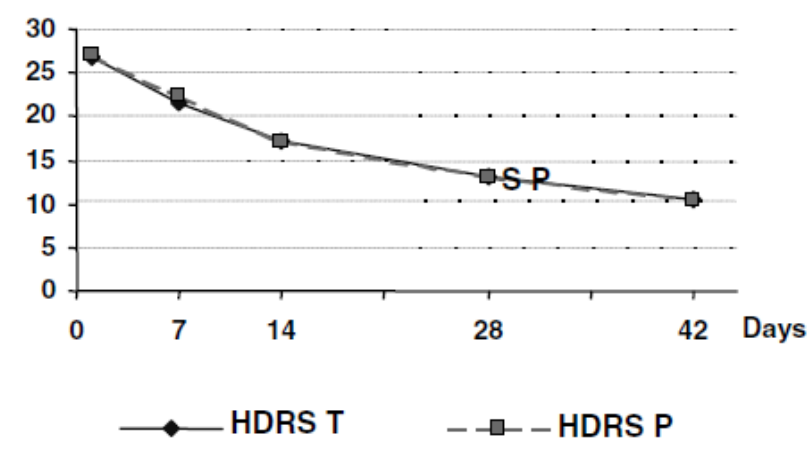

Figure 2. Evolution of HDRS global score (PP population)

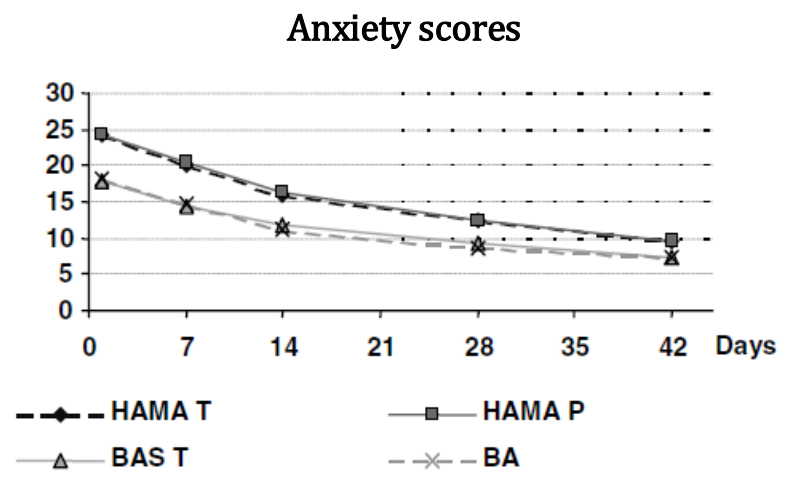

Figure 3. Evolution of anxiety scales (PP population)

\section{TOLERABILITY}

Fifty-eight patients (18\%) withdrew from the study, 29 in the tianeptine group and 29 in the paroxetine group ( $p=0.940)$. However, reasons for withdrawal were significantly different in the two groups $(p=0.001)$, with more withdrawal for adverse event (19 versus 6$)$ in the paroxetine group versus more withdrawals for lack of efficacy (11 versus 4 ) or non-medical reason (11 versus 4 ) in the tianeptine group. One patient withdrew due to recovery in the tianeptine group and two patients were lost to follow up in the paroxetine group. At inclusion, only minor and non-significant laboratory abnormalities were reported for recruited patients. As the two study products are well-known drugs, no further laboratory measures were taken.

Tolerability of the two products was good. During the whole study period (including the inclusion visit), 204 patients (62\%) reported at least one adverse event, 96 (59\%) in the tianeptine group and 108 (65\%) in the paroxetine group.

Emergent adverse events were reported in 152 patients (46\%), with a significantly higher frequency ( $p=0.026$ ) in the paroxetine group (87 patients, 53\%) than in the tianeptine group (65 patients, 40\%). The most frequently reported emergent adverse events were nausea (7\%) and headaches (6\%) in the tianeptine group versus nausea (15\%), headaches (8\%), insomnia (6\%) and dizziness (5\%) in the paroxetine group (Table 4). This was confirmed using the 
AMDP-5 check list where the most frequent emergent symptoms were nausea $(T=7 \%, P=$ $15 \%)$ and headaches $(T=10 \%, P=10)$.

Severe, treatment-related adverse events, despite a low overall incidence, were also more frequent in the paroxetine group (seven events: two nausea, headache, malaise, weight increase, abdominal pain and bradycardia) than in the tianeptine group (only two events: headache and abdominal pain).

Despite the lack of a dose-tapering procedure, no withdrawal syndrome was reported after discontinuation of either drug. However, a post visit was not performed for all the patients and, when feasible, was at any time between the end of the 6-week double-blind period and the end of the optional continuation period (up to 6 months). Utilising only post visits performed 1 week after D42 in patients without immediate switch from the study drug to another antidepressant, permitted analysis of barely more than half of the patients (tianeptine: $n=82$; paroxetine: $n=$ 90). For these patients, CGI scores were similar at post visit and complement CHESS did not show any major discrepancies in somatic symptoms present 7 days after withdrawal.

Table 4. Most frequent emergent adverse events

\begin{tabular}{lcc}
\hline Symptom & Tianeptine $(n=162)$ & Paroxetine $(n=165)$ \\
\hline & & \\
Overall* & $65(40 \%)$ & $87(53 \%)$ \\
Nausea & $11(7 \%)$ & $24(15 \%)$ \\
Headache & $10(6 \%)$ & $14(8 \%)$ \\
Insomnia & $2(1 \%)$ & $10(6 \%)$ \\
Dizziness & $1(1 \%)$ & $8(5 \%)$ \\
\hline
\end{tabular}

${ }^{*} p=0.02$ ( $\mathrm{p}$-values not assessed for individual symptoms).

\section{Discussion}

This trial was intended to study both the depressive and the anxious components of major depressive disorder. Methodological problems were outlined in Section 1. Only moderate to severe depressed patients, according to DSM-IV criteria, were included: a minimum rating of 25 on MADRS was required. As depressive scales, and especially the HDRS, also include anxious symptoms, a sub-scale of HDRS (items 1, 2, 7, 8, 10 and 13) presumed to be more specific for depressive symptoms was also used with a minimum threshold of 9 . Thus, only patients with an authentic Major Depression participated in the study.

In order to limit co-morbid anxious disorders, special attention was given at inclusion to psychiatric and therapeutic antecedents. It was decided not to include patients with a previous diagnosis of any characterised anxious disorder except General Anxiety Disorder (GAD), but only if not within the last year. Moreover, to avoid undiagnosed anxious disorders or severe former GAD, major restrictions were made on previous use of anxiolytic treatments, and especially benzodiazepines (time of prescription and maximum doses).

Despite these strict exclusions regarding previous or comorbid anxiety disorders, anxiety scores at inclusion remained high, as expected when designing the protocol. These restrictions do not 
guarantee that no patient suffered previous or concomitant anxious disorder, or that no patient later developed a defined anxious disorder, but limit these possibilities both in frequency and in severity.

Thus, patients in the trial can be assumed to be as 'pure depressive' as possible, and anxious symptoms can be attributed more to the depressive illness itself than to a possible co- or premorbid anxious pathology.

The first objective of the study was to compare tianeptine and paroxetine with regard to their antidepressant efficacy. The present study demonstrates that both tianeptine and paroxetine are effective treatments at 6 weeks in patients suffering from major depression without defined comorbid anxious disorder.

Scores on the different depression scales were very similar throughout the study and no difference in efficacy appears between the two treatments. Response rates were high in the two groups, with more than $60 \%$ of responders whatever the treatment or the evaluation scale (MADRS or HDRS), without a significant difference between groups.

The second objective of the study was to evaluate in this population the effectiveness of tianeptine and paroxetine on anxiety. Despite the rigour of the exclusion criteria regarding comorbid anxious disorders, mean initial inclusion scores on anxiety scales were quite high (24 for HAMA and 18 for BAS), which confirms the importance of anxious symptoms in depressive syndrome mentioned in the literature (Hamilton, 1989; Kuhs, 1991). At the end of the study, improvements on anxiety scale were highly significant, without any observed difference between treatments.

It was assumed before the trial that depression scores and anxiety scores would be highly correlated, making the usual anxiety instruments of low accuracy for the estimation of anxiety relief in depression (Riskind et al., 1987; Wetzler and Katz, 1989; Watson et al., 1995a,b). This was not confirmed in this study. However, the independence between scales was not fully demonstrated (especially for the two Hamilton scales) and the planned factorial analysis was conducted to isolate possibly more specific clusters for depression and anxiety. This analysis is not yet complete and the results regarding this part of the study will be presented separately in extenso.

Briefly, correlation analysis between anxiety scales and depression scales at D1 were undertaken and the coefficients found were rather low, except for the two Hamilton scales (HDRS and HAMA) where it was $>0.5$. The predictive value of anxiety scales on final MADRS score was also poor, and covariance analysis never reached statistical significance.

The poor correlations observed between anxiety scales and depression scales at inclusion suggest that the observed improvement of anxiety is, at least partly, related to actions of the treatments on the anxious dimension of the pathology and not merely to an indirect effect linked to the improvement of depressive symptoms. As both tianeptine and paroxetine are known effective antidepressant drugs and also usually have a positive action on anxious symptoms, this lack of observed difference for both depression and anxiety scales is not surprising.

The tolerability of the two drugs was quite good. The most common adverse events were general (mainly headaches) and gastro-intestinal (mainly nausea), consistent with the known side-effect 
profiles of the two drugs. Very few adverse events were considered severe and none had serious consequences for the patients. However, tolerability of tianeptine was slightly but significantly better than paroxetine, with less withdrawal for adverse events, less emergent adverse events and less severe treatment-related adverse events.

No withdrawal symptoms after cessation of tianeptine or paroxetine treatment were observed in this study. However, methodological problems in the design of the post study visit (only half of the patients assessable) limit the validity of this observation.

\section{Conclusion}

Both $37.5 \mathrm{mg}$ a day of tianeptine and $20 \mathrm{mg}$ a day of paroxetine are safe and effective treatments for patients suffering from major depression. Improvements are not only observed in depressive symptoms, but also in anxious symptoms with both treatments. Despite onerous requirements regarding patient selection, control of co-prescriptions and assessment methods, it was not possible to demonstrate whether the improvement in anxious symptoms was linked to the general improvement of depressive pathology or was, at least partially, due to a specific action of the drugs on anxiety. However, the limited individual correlations between initial anxiety symptoms and initial and final depressive symptoms suggest that the anxiety scales used in the study are valid tools for this population. It thus appears that both tianeptine and paroxetine could have a direct action on anxiety in depressive illness, independently of their overall influence on depression itself. However, due to the lack of a placebo control arm, such inferences are only tentative and need confirmation. 


\section{References}

Alby JM, Cabane J, Ferreri M, Bougerol T. 1993. Efficacy and tolerability of tianeptine in major depressive disorder and in dysthymia (DSM III R) with somatic complaints: double blind study versus fluoxetine. Eur Neuropsychopharmacol 3(3): 333.

American Psychiatric Association. 1994. Diagnostic and Statistical Manual of Mental Disorders (4th edn). American Psychiatric Association: Washington, DC.

Andreasen NC, Grove WM, Maurer R. 1980. Cluster analysis and the classification of depression. $\mathrm{Br}$ J Psychiatry 137: 256-265.

de Bonis M, Lebeaux MO, de Boeck P, Simon M, Pichot P. 1991. Measuring the severity of depression through a self-report inventory. A comparison of logistic, factorial and implicit models. J Affect Disord 22: 55-64.

Boulenger JP, Fournier M, Rosales D, Lavallée Y-J. 1997. Mixed anxiety and depression: from theory to practice. J Clin Psychiatry 58(Suppl. 8): 27-34.

Boyer WF, Feighner JP. 1992. An overview of paroxetine. J Clin Psychiatry 53(Suppl. 2): 3-6.

Cassano GB, Heinze G, LAoo H, Mendlewicz J, Paes de Sousa M. 1996. A double-blind comparison of tianeptine, imipramine and placebo in the treatment of major depressive episodes. Eur Psychiatry 11: 254-259.

Clark LA, Watson D. 1991. Tripartite model of anxiety and depression: psychometric evidence and taxonomic implications. J Abnorm Psychol 100(3): 316-336.

Costa e Silva JA, Ruschel S, Caetano D, et al. 1997. Placebo-controlled study of tianeptine in major depressive episodes. Neuropsychobiology 35: 24-29.

Dalery J, Dagens-Lafont V, de Bodinat C. 2001. Efficacy of tianeptine vs placebo in the long term treatment (16.5 months) of unipolar major recurrent depression. Hum Psychopharmacol: Clin Exp 16(Suppl. 1), S39S47.

Fawcett J. 1997. The detection and consequences of anxiety in clinical depression. J Clin Psychiatry 58(Suppl. 8): 35-40.

Foster RH, Goa KL. 1997. Paroxetine. A review of its pharmacology and therapeutic potential in the management of Panic Disorder. CNS Drugs 8: 163-188.

Guelfi JD, Pichot P, Dreyfus JF. 1989. Efficacy of tianeptine in anxious-depressed patients: results of a controlled multicenter trial versus amitriptyline. Neuropsychobiology 22: 41-48.

Hamilton M. 1989. Frequency of symptoms in melancholia (depressive illness). Br J Psychiatry 154: 201206.

Joffe RT, Bagby M, Levitt A. 1993. Anxious and nonanxious depression. Am J Psychiatry 150: 1257-1258.

Kessler RC, Nelson CB, McGonagle KA, et al. 1996. Comorbidity of DSM-III-R depressive disorder in the general population: results from the US National Comorbidity Survey. Br J Psychiatry 168(Suppl. 30): 1730.

Kravitz HM, Fogg L, Fawcett J, Edwards J. 1989. Antidepressant or antianxiety? A study of the efficacy of antidepressant medication. Psychiatry Res 32: 141-149.

Kuhs H. 1991. Anxiety in depressive disorders. Compr Psychiatry 32: 217-228. 
Lépine JP. 1994. Comorbidité des troubles anxieux et dépressifs: perspectives épidémiologiques. Encéphale XX: 683-692.

Lôo H, Malka R, Defrance R, et al. 1988. Tianeptine and amitriptyline: controlled double-blind trial in depressed alcoholic patients. Neuropsychobiology 19: 79-85.

Lôo H, Saiz-Ruiz J, Costa e Silva JA, et al. 1999. Efficay and safety of tianeptine in the treatment of depressive disorders in comparison with fluoxetine. J Affect Disord 56: 109-118.

Maier W, Falkai P. 1999. The epidemiology of comorbidity between depression, anxiety disorders and somatic diseases. Int Clin Psychopharmacol 14(Suppl. 2): S1-6.

Martinsen EW, Friis S, Hoffart A. 1989. A factor analytical study of the comprehensive psychopathological rating scale among patients with anxiety and depressive disorders. Acta Psychiatr Scand 80: 492-498.

Riskind JH, Beck AT, Brown G, Steer RA. 1987. Taking the measure of anxiety and depression. Validity of the reconstructed Hamilton scales. J Nerv Ment Dis 175(8): 474-479.

Sartorius N, Üstün TB, Lecrubier Y, et al. 1996. Depression comorbid with anxiety: results from the WHO study on psychological disorders in primary health care. Br J Psychiatry 168(Suppl. 30): 38-43.

Watson D, Clark LA, Weber K, Assenheimer JS, Strauss ME, McCormick RA. 1995a. Testing a tripartite model. I. Evaluating the convergent and discriminant validity of anxiety and depression symptom scales. J Abnorm Psychol 104: 3-14.

Watson D, Clark LA, Weber K, Assenheimer JS. 1995b. Testing a tripartite model. II. Exploring the symptom structure of anxiety and depression in student, adult, and patient samples. J Abnorm Psychol 104(1): 15-25.

Wetzler S, Katz MM. 1989. Problems with the differentiation of anxiety and depression. J Psychiatr Res 23: $1-12$.

Wittchen HU, Schuster P, Lieb R. 2001. Comorbidity and mixed anxiety-depressive disorder (MAD): clinical curiosity or pathophysiological need? Hum Psychopharmacol: Clin Exp 16(Suppl. 1), S21-S30.

Wittchen HU, Zhao S, Kessler RC, Eaton WW. 1994. DSM-III-R generalized anxiety disorder in the national comorbidity survey. Arch Gen Psychiatry 51: 355-364.

World Health Organization. 1993. The ICD-10 Classification of Mental and Behavioural Disorders. Diagnostic Criteria for Research. World Health Organization: Geneva, F41.2. 